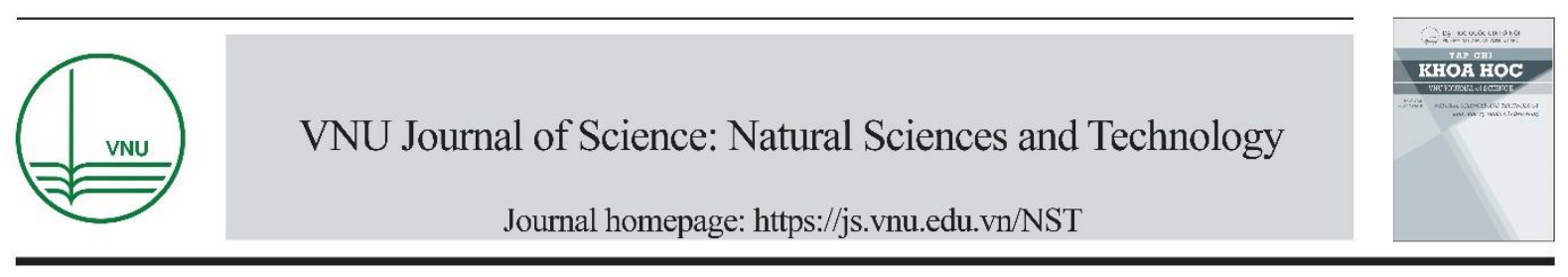

Original Article

\title{
The Structure and Crystallization Process of Amorphous Iron Nanoparticles
}

\author{
Duong Thi Thanh, Pham Mai An, Giap Thi Thuy Trang, \\ Nguyen Thi Minh Thuy, Pham Huu Kien* \\ Thai Nguyen University of Education, 28 Luong Ngoc Quyen, Thai Nguyen, Vietnam
}

Received 02 May 2019

Revised 16 May 2019; Accepted 17 May 2019

\begin{abstract}
This paper studies the crystallization process and structure of amorphous iron nanoparticles by molecular dynamics method. The study shows that amorphous iron nanoparticles could not be crystallized at $300 \mathrm{~K}$ and $500 \mathrm{~K}$. Iron nanoparticle, annealed at $900 \mathrm{~K}$ over a long time, was crystallized into a BCC crystal structure. The structure of crystallized iron nanoparticle at 900 $\mathrm{K}$ was analyzed through the pair radial distribution function and the number of crystal atoms upon various regions in nanoparticles. The simulation revealed that the first nuclei was formed most frequently in the area near the surface of the nanoparticle. Then the crystal cluster grew toward the centre of the nanoparticle. The completely crystallized nanoparticle had two components: the core with a BCC crystal structure and surface with an amorphous structure. As for the amorphous nanoparticle at 300 or $500 \mathrm{~K}$, crystal-clusters were too small to grow large enough to crystallize the nanoparticle.
\end{abstract}

Keywords: Iron nanoparticle, crystallize, annealing, crystal atom, crystal cluster.

\footnotetext{
* Corresponding author.

Email address: phamhuukien@dhsptn.edu.vn

https://doi.org/10.25073/2588-1140/vnunst.4891
} 


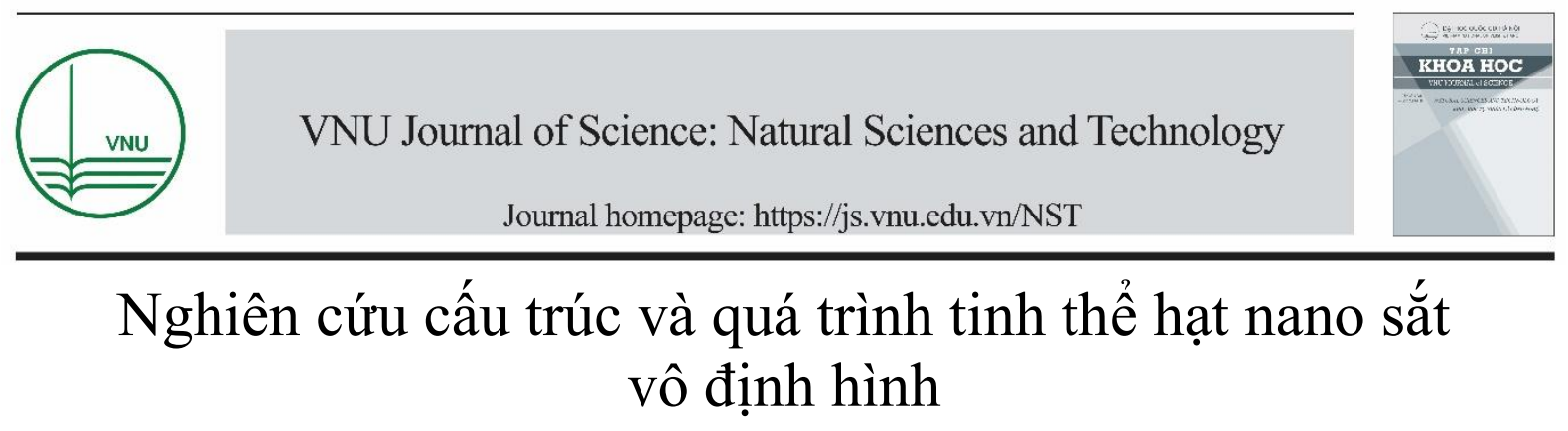

\author{
Dương Thị Thanh, Phạm Mai An, Giáp Thị Thùy Trang, \\ Nguyễn Thị Minh Thủy, Phạm Hữu Kiên* \\ Truờng Đại học Su phạm, Đại học Thái Nguyên, 20 Luoong Ngọc Quyến, TP. Thái Nguyên, Việt Nam \\ Nhận ngày 02 tháng 5 năm 2019 \\ Chỉnh sửa ngày 16 tháng 5 năm 2019; Chấp nhận đăng ngày 17 tháng 5 năm 2019
}

\begin{abstract}
Tóm tắt: Trong bài báo này, chúng tôi nghiên cứu cấu trúc và quá trình tinh thể hạt nano sắt vô định hình (VĐH) bằng phương pháp mô phỏng động lực học phân tử (ĐLHPT). Chúng tôi đã phát hiện các hạt nano sắt ở nhiệt độ $300 \mathrm{~K}$ và $500 \mathrm{~K}$ không thể tinh thể. Trong khi đó đối với hạt nano sắt ở $900 \mathrm{~K}$, sau thời gian hồi phục $2 \times 10^{7}$ bước, nó tinh thể thành cấu trúc bcc. Cấu trúc hạt nano tinh thể sắt ở $900 \mathrm{~K}$ được phân tích thông qua hàm phân bố xuyên tâm (HPBXT), số nguyên tử tinh thể theo các lớp khác nhau trong hạt nano. Mô phỏng chỉ ra rằng, ban đầu các nhân tinh thể được tạo thành chủ yếu trong vùng gần bề mặt hạt nano. Sau đó các đám tinh thể phát triển theo hướng tới tâm hạt nano. Cuối cùng hạt nano tinh thể có hai phần: lõi với cấu trúc tinh thể bcc và bề mặt với cấu trúc VĐH. Với hạt nano sắt ở 300 và $500 \mathrm{~K}$, do các đám tinh thể nhỏ nên chúng không thể phát triển tới kích thước tới hạn để có thể tinh thể hạt nano.
\end{abstract}

Tù khóa: Hạt nano sắt, tinh thể, ủ nhiệt, nguyên tử tinh thể, đám tinh thể.

\section{Giới thiệu}

Hạt nano có thể tạo được ở trạng thái tinh thể hoặc vô định hình (VĐH) bằng các phương pháp tổng hợp khác nhau [1-3]. Hạt nano VĐH có cấu trúc mất trật tự, nó được chia thành hai phần: lõi với các đặc trưng cấu trúc tương tự dạng khối VĐH của vật liệu tương ứng và bề mặt có cấu trúc VĐH xốp hơn. Vì cấu trúc đặc biệt mà hạt nano có nhiều ứng dụng quan trọng trong các lĩnh vực khác nhau của khoa học và công nghệ $[4,5]$. Chẳng hạn như, trong các chất xúc tác, hạt nano $\mathrm{Fe}_{2} \mathrm{O}_{3} \mathrm{VĐH}$ linh động hơn so với nano

\footnotetext{
* Tác giả liên hệ.

Địa chi email: phamhuukien@dhsptn.edu.vn

https://doi.org/10.25073/2588-1140/vnunst.4891
}

$\mathrm{Fe}_{2} \mathrm{O}_{3}$ tinh thể ở cùng kích thước, hình dạng [4]. Nói chung, trạng thái VĐH của hạt nano không bền vững và hạt nano dễ bị tinh thể khi được ủ nhiệt. Sự ổn định của hạt nano VĐH ngăn cản sự tinh thể đang rất được quan tâm, vì nó liên quan đến khả năng làm việc của chúng trong thực tế. Đến bây giờ sự tinh thể của hạt nano VĐH chủ yếu được nghiên cứu bằng thực nghiệm. Ví dụ, sự chuyển pha trong hạt nano $\mathrm{V} Đ H$ coban được nghiên cứu bởi các đường cong DSC (Differential Scanning Calorimetry). Nhiệt độ chuyển thủy tinh và nhiệt độ tinh thể của hạt nano $\mathrm{VĐH}$ đã được phát hiện là phụ thuộc vào 
kích thước [5]. Một số công trình [6-8] đã được thực hiện với sự tinh thể của hạt nano $\mathrm{TiO}_{2} \mathrm{VĐH}$. Hạt nano này biến đổi thành trạng thái 'anatase' và sau đó thành 'rutile' phụ thuộc vào nhiệt độ hồi phục hoặc điều kiện chế tạo. Chúng tôi cũng tìm thấy một vài công trình thực nghiệm liên quan đến sự biến đổi VĐH-tinh thể của hạt nano $\mathrm{Al}_{2} \mathrm{O}_{3}$ và $\mathrm{CdSe}[9,10]$. Quá trình tinh thể có thể được nghiên cứu bằng phương pháp mô phỏng vì nó rất khó để theo dõi trực tiếp chuyển động của nguyên tử bằng thực nghiệm. Trong bài báo này, chúng tôi thực hiện mô phỏng quá trình biến đổi cấu trúc hạt nano $\mathrm{Fe} \mathrm{V} Đ H$ ở các nhiệt độ hồi phục khác nhau để làm rõ hơn quá trình tinh thể và cấu trúc của hạt nano tinh thể Fe.

\section{Phương pháp mô phỏng}

Phương pháp động lực học phân tử (ĐLHPT)

$$
U\left(r_{i j}\right)=-0,188917\left(r_{i j}-1,82709\right)^{4}+0,170192\left(r_{i j}-2,50849\right)^{2}-0,198294, r_{i j}<3,44 \AA
$$

Ở đây, $r_{i j}$ là khoảng cách tương tác giữa nguyên tử thứ $i$ và thứ $j, r_{i j}$ có đơn vị là $\AA$, $U(r)$ có đơn vị $\mathrm{eV}$. Hạt nano được hồi phục bằng phương pháp thống kê hồi phục (hay phương pháp động lực học phân tử đối với hệ có nhiệt độ bằng không) cho đến khi hệ đạt được trạng thái cân bằng (năng lượng của hệ không đổi theo thời gian mô phỏng, với hạt nano Fe năng lượng trên nguyên tử ổn định bằng $-1,2844 \mathrm{eV}$ sau thời gian mô phỏng khoảng $10^{5}$ bước). Sau đó, hạt nano được đun nóng đến các nhiệt độ 300 $\mathrm{K}, 500 \mathrm{~K}$ và $900 \mathrm{~K}(900 \mathrm{~K}$ là điểm gần nhiệt độ thủy tinh $T_{g} \approx 1070 \mathrm{~K}$ [12]). Ở mỗi nhiệt độ trên, hệ được ủ nhiệt bằng phương pháp ĐLHPT với số hạt, áp suất phòng và nhiệt độ không đổi (ủ nhiệt NPT) sau khoảng $2 \times 10^{7}$ bước mô phỏng (thời gian 1 bước mô phỏng bằng $4,12 \mathrm{fs}$ ). Bằng phương pháp trên, chúng tôi nhận được các hạt nano $\mathrm{Fe}$ tương ứng với nhiệt độ $300 \mathrm{~K}, 500 \mathrm{~K}$ và 900 K. Khác với mẫu Fe khối, đối với hạt nano Fe có dạng hình cầu, HPBXT $g_{\text {nano }}(r)$ được xác định bởi: được sử dụng để nghiên cứu cấu trúc và quá trình tinh thể hạt nano Fe VĐH. Ban đầu 5000 nguyên tử Fe được gieo ngẫu nhiên trong một quả cầu (hạt nano) có bán kính sao cho, mật độ khối lượng của hạt nano nhỏ hơn "không quá nhiều" mật độ khối lượng của mẫu Fe lỏng $(7,01$ g.cm-3), do đó bán kính ban đầu của hạt nano có thể chọn bằng $27 \AA$ Å, với điều kiện biên tự do (khi sử dụng biên tự do, sau thời gian ủ nhiệt thích hợp, hạt nano đạt được trạng thái có mật độ xác định). Trong bài báo, chúng tôi chọn thế tương tác là thế cặp Pak-Doyama, vì tính đơn giản và thực tế cho thấy việc sử dụng thế tương tác cặp PakDoyama cho phép mô phỏng các vật liệu kim loại $(\mathrm{Fe}, \mathrm{Ni}, \mathrm{Co}, \ldots)$ có nhiều tính chất phù hợp với số liệu thực nghiệm như HPBXT hoặc thừa số cấu trúc, phân bố số phối trí...[11, 12]. Thế tương tác cặp Pak-Doyama đối với hạt nano $\mathrm{Fe}$ có dạng:

$$
g_{\text {nano }}(r)=\frac{\left\langle n_{\text {nano }}(r)\right\rangle}{\left\langle V_{\text {in }}\right\rangle \rho_{\text {nano }}}
$$

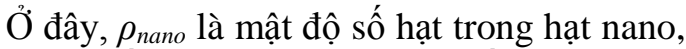
$n_{\text {nano }}(r)$ là số nguyên tử trong lớp cầu có độ dày $d r$ ở khoảng cách $r$ tính từ nguyên tử tâm, $V_{i n}$ và $V_{\text {out }}$ là thể tích phần trong và ngoài của lớp cầu, ở đây $V_{i n}+V_{\text {out }}=4 \pi r^{2} d r$. Dấu 〈 〉là ký hiệu tính trung bình. Hạt nano cầu gồm lõi với mật độ bằng mật độ của mẫu khối và bề mặt có mật độ thấp hơn. Nếu tất cả các nguyên tử trong vùng bề mặt được chuyển đi, chúng tôi nhận được lõi hạt nano. Trong mô phỏng hiện tại, cấu trúc hạt nano được phân tích thông qua HPBXT của lõi.

Trong tính toán, chúng tôi xác định nguyên tử tinh thể và nguyên tử VĐH như sau: Nguyên tử gọi là nguyên tử tinh thể có cấu trúc bcc nếu chúng thỏa mãn hai điều kiện: i) có 14 lân cận; ii) có 6 nguyên tử trong số 14 lân cận có 4 nguyên tử lân cận chung (tương ứng với vị trí cực đại thứ hai trong HPBXT của mẫu Fe có cấu trúc tinh thể bcc) và 8 nguyên tử có 6 nguyên tử lân cận chung với nguyên tử tâm (tương ứng với 
vị trí cực đại thứ nhất trong HPBXT của mẫu Fe có cấu trúc tinh thể bcc). Ở đây bán kính ngắt xác định nguyên tử lân cận bằng $3,35 \AA$ (bán kính ngắt được xác định như là vị trí cực tiểu thứ nhất trong HPBXT đối mẫu sắt khối ở $300 \mathrm{~K}$ [11,12]). Các nguyên tử còn lại trong hạt nano gọi là các nguyên tử $\mathrm{VĐH}$. Để thuận tiện, gọi $N$ là tổng số nguyên tử; $N_{A}, N_{C r}$ lần lượt là số nguyên tử $\mathrm{VĐH}$ và tinh thể. Đám tinh thể nhỏ nhất chỉ có 15 nguyên tử bao gồm nguyên tử trung tâm và 14 lân cận của nó. Khi đám tinh thể tăng thì $N_{C r}$ tăng còn $N_{A}$ sẽ giảm. Hạt nano có hai loại nguyên tử tinh thể. Loại thứ nhất (nguyên tử tinh thể bề mặt) liên quan đến nguyên tử có lân cận gồm cả nguyên tử tinh thể và VĐH. Loại hai (nguyên tử

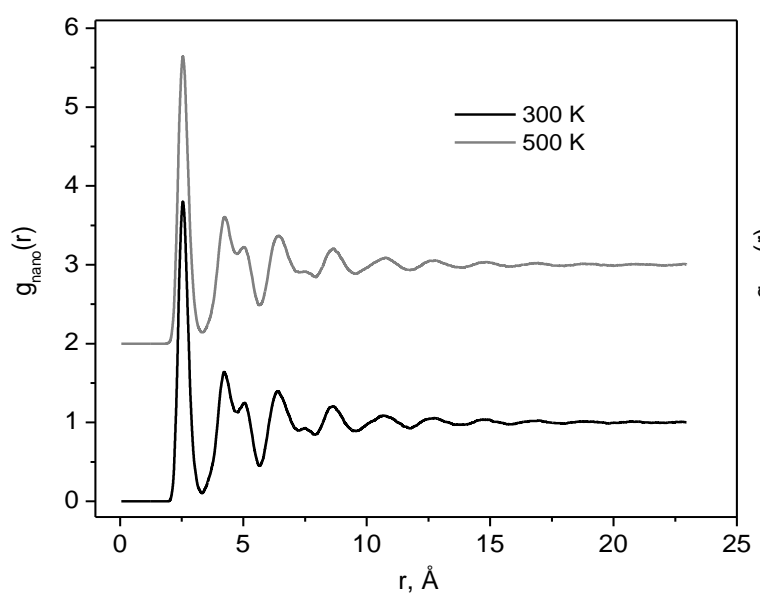

Hình 1. HPBXT lõi hạt nano ủ nhiệt ở nhiệt độ $300 \mathrm{~K}$ và $500 \mathrm{~K}$.

Hình 2 cho thấy HPBXT lõi hạt nano $\mathrm{Fe}$ ở $900 \mathrm{~K}$ sau khi hồi phục $1 \times 10^{6}$ bước và $2 \times 10^{7}$ bước mô phỏng. Có thể thấy trên hình 2 , HPBXT thay đổi mạnh trong suốt thời gian hồi phục. Khi thời gian hồi phục ngắn $\left(1 \times 10^{6}\right.$ bước $)$, đường cong HPBXT tương tự như HPBXT Fe khối $\mathrm{VĐH}$. Trong khoảng thời gian này, hạt nano $\mathrm{Fe}$ vẫn có cấu trúc $\mathrm{VĐH,} \mathrm{nghĩa} \mathrm{là} \mathrm{chưa} \mathrm{có} \mathrm{đám} \mathrm{tinh}$ thể nào xuất hiện trong các vùng thể tích của hạt nano. Như thấy trên hình 2 , khi thời gian ủ nhiệt dài hơn (sau $2 \times 10^{7}$ bước), thì HPBXT của hạt nano $\mathrm{Fe}$ xuất hiện nhiều cực đại thứ cấp, nghĩa là HPBXT cho thấy các đặc trưng của cấu trúc tinh tinh thể lõi) có lân cận chỉ là nguyên tử tinh thể.

\section{Kết quả và thảo luận}

Hình 1 cho thấy HPBXT của lõi hạt nano 300 $\mathrm{K}$ và $500 \mathrm{~K}$. Có thể thấy rằng đường cong ở nhiệt độ 300 và $500 \mathrm{~K}$ có một đỉnh chính đặt tại điểm $2,25 \AA$ và đỉnh thứ 2 tách thành hai đỉnh nhỏ, đỉnh nhỏ bên trái cao hơn đỉnh nhỏ bên phải. Đặc điểm này của HPBXT cho thấy đặc trưng cấu trúc của kim loại $\mathrm{Fe}$ VĐH. HPBXT cũng cho thấy sự tương tự với Fe khối như được báo cáo trong các công trình $[11,12]$. Vậy, lõi hạt nano Fe VĐH có cấu trúc tương tự Fe khối VĐH.

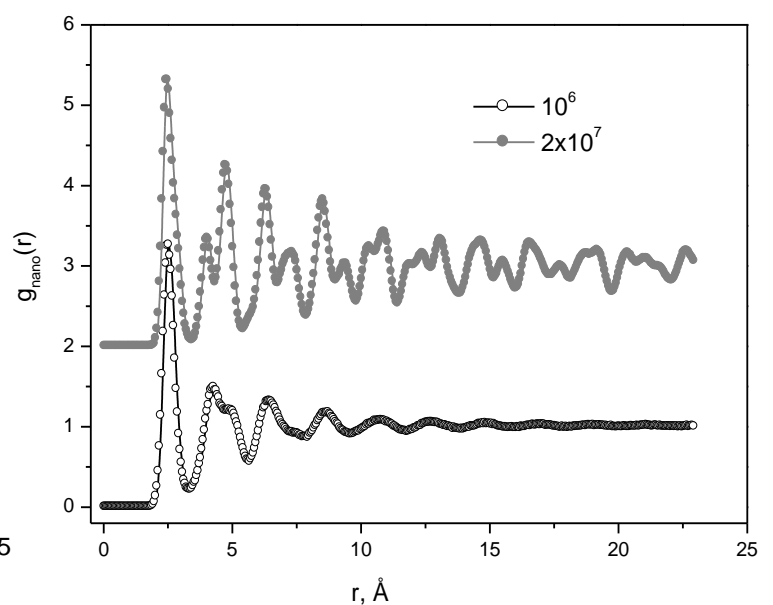

Hình 2. HPBXT lõi hạt nano ủ nhiệt ở $900 \mathrm{~K}$ sau khoảng thời gian $10^{6}$ và $2 \times 10^{7}$ bước.

thể (khác với mẫu VĐH, độ cao cực đại chính (đỉnh thứ nhất) của HPBXT lớn hơn và rõ ràng hơn nhiều so với một vài cực đại còn lại, tức là HPBXT thể hiện các đặc trưng của cấu trúc trật tự gần). Vị trí, hình dạng các đỉnh của HPBXT sau khoảng thời gian $2 \times 10^{7}$ bước là bằng chứng có sự biến đổi từ cấu trúc VĐH sang cấu trúc tinh thể.

Thông tin về quá trình biến đổi hạt nano Fe VĐH sang hạt nano $\mathrm{Fe}$ tinh thể bcc được khảo sát thông qua sự thay đổi số các nguyên tử tinh thể bcc trong quá trình ủ nhiệt NPT hạt nano $\mathrm{Fe}$ ở $900 \mathrm{~K}$ (xem hình 3 ). Hình 3 chỉ ra rằng, quá trình 
biến đổi cấu trúc có thể chia thành 3 giai đoạn. Giai đoạn thứ nhất (sau $2 \times 10^{6}$ bước), số nguyên tử tinh thể tăng không đáng kể, ở giai đoạn này, mặc dù đám tinh thể xuất hiện nhưng chúng không bền vững. Trong khi đó giai đoạn thứ hai (trong khoảng $2 \times 10^{6}$ bước đến $5 \times 10^{6}$ bước), số nguyên tử tinh thể tăng đột ngột. Trong giai đoạn hai đám tinh thể xuất hiện bền vững hơn và kích thước của chúng lớn dần theo thời gian ủ nhiệt. Điều này chứng tỏ rằng, nếu kích thước của đám tinh thể lớn hơn một giá trị tới hạn, thì đám tinh thể có khả năng tăng đột ngột và đạt đến một kích thước cực đại. Chúng tôi xác định được giá trị tới hạn của đám tinh thể là khoảng 230 - 300 nguyên tử tinh thể. Giai đoạn thứ ba, số nguyên tử tinh thể dao động quanh một giá trị nhất định. Trong giai đoạn này, kích thước của đám tinh thể đã đạt đến một giá trị cực đại (giá trị bão hòa). Nghĩa là, hạt nano Fe VĐH đã tinh thể hoàn toàn trong giai đoạn ba. Phân tích trên cho thấy, quá trình tinh thể hạt nano $\mathrm{Fe} V \mathrm{VH}$ thành câu trúc bcc rất phù hợp tốt với lý thuyết nhân tinh thể cổ điển [10].

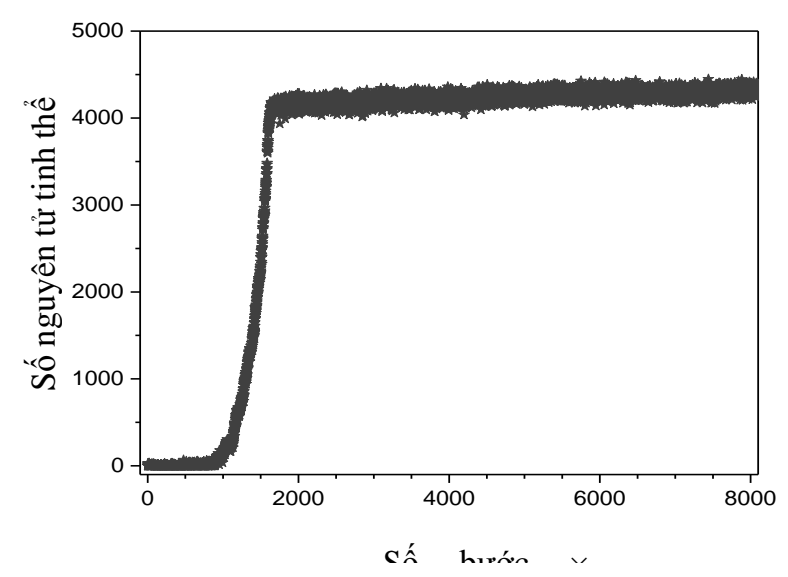

Hình 3. Phân bố số nguyên tử tinh thể theo thời gian.
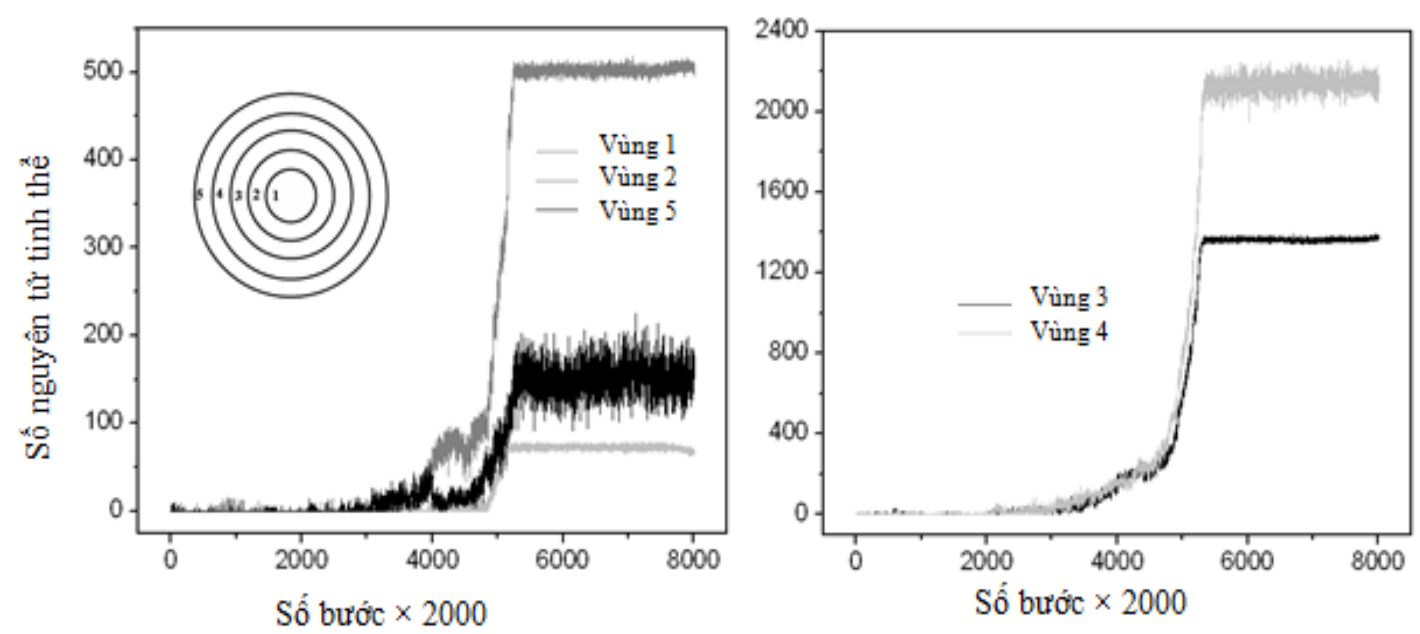

Hình 4. Phân bố số nguyên tử tinh thể trong năm vùng của hạt nano theo thời gian. 
Để hiểu rõ hơn về quá trình hình thành và phát triển của các đám tinh thể trong hạt nano $\mathrm{Fe}$ VĐH ở $900 \mathrm{~K}$, chúng tôi chia hạt nano này thành 5 vùng khác nhau như được minh họa trên hình bên trong của hình 4 . Ở đây, vùng 1 là một quả cầu có bán kính 5,4 Å. Các vùng còn lại là các lớp cầu có độ dày liên tiếp bằng $5,4 \AA$. Như thấy trên hình 4 , sau thời gian $8 \times 10^{6}$ bước mô phỏng đầu tiên, có một vài nhân với kích thước nhỏ hơn 50 nguyên tử được tạo thành trong tất cả các vùng thể tích của hạt nano và biến mất theo thời gian hồi phục. Trong suốt thời gian $2 \times 10^{6}$ bước tiếp theo, đám tinh thể mở rộng chủ yếu trong vùng 3 và 4 , trong khi vùng 1 và 5 tăng rất chậm.
Quá trình tăng cũng xảy ra trong vùng 2 nhưng với tốc độ chậm hơn nhiều so với vùng 3 và 4 . Khi thời gian hồi phục tăng từ $2 \times 10^{6}$ bước đến $10^{7}$ bước mô phỏng, đám tinh thể phát triển đột ngột trên toàn thể tích hạt nano. Quá trình tăng trong vùng 4 xảy ra với tốc độ mạnh nhất và chậm dần trong các vùng $3,2,5$ và 1 . Sau thời gian $10^{7}$ bước, số nguyên tử tinh thể trong tất cả các vùng đạt trạng thái bão hòa và dao động quanh những giá trị nhất định. Lúc này, sự biến đổi lớn nhất xảy ra trong vùng 5 . Sự biến đổi mạnh trong vùng 5 được cho là do các nguyên tử trong vùng 5 (tức là vùng bề mặt) sẽ linh động hơn nhiều so với các vùng khác.
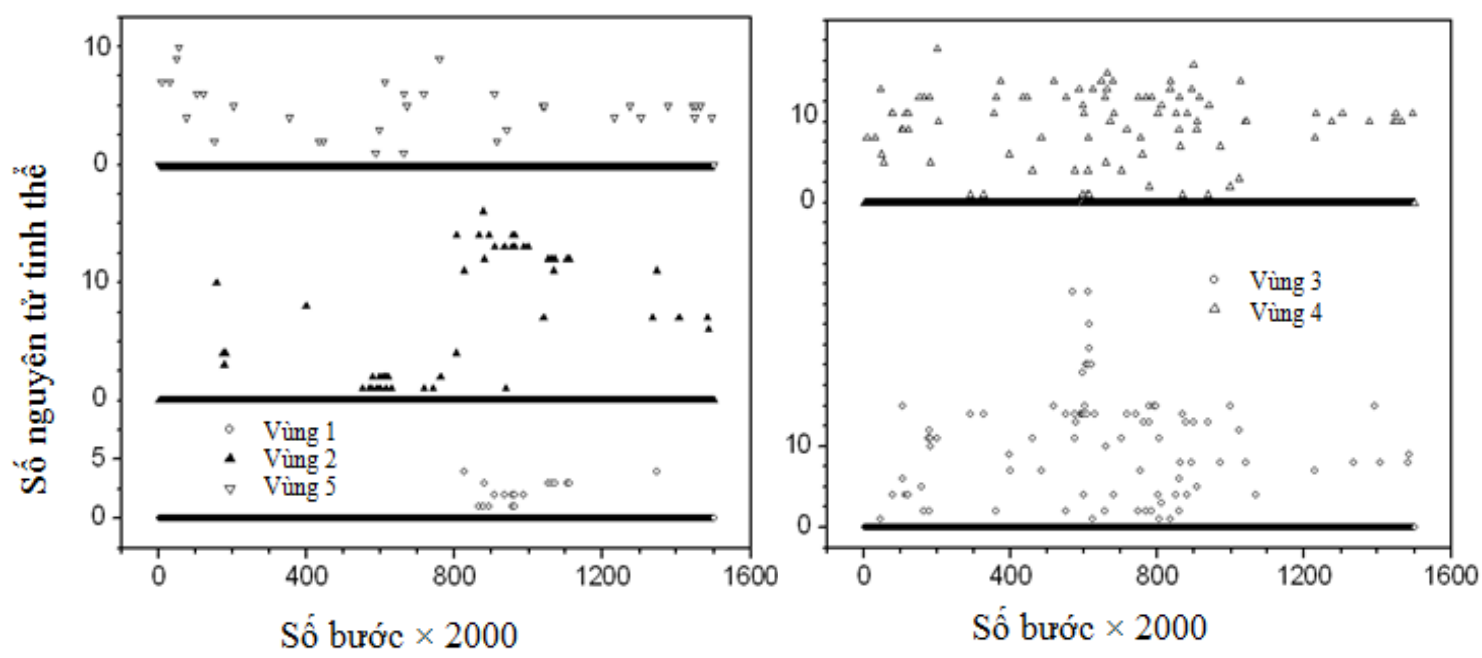

Hình 5. Phân bố số nguyên tử tinh thể trong năm vùng của hạt nano trong khoảng thời gian $3 \times 10^{6}$ bước mô phỏng đầu tiên.

Để trực quan chi tiết hơn về quá trình tinh thể trong hạt nano $\mathrm{Fe}$, chúng tôi thực hiện vẽ sự phát triển của các nhân tinh thể trong $3 \times 10^{6}$ bước mô phỏng đầu tiên. Như thấy trên hình 5 , những nhân nhỏ là không ổn định để có thể hình thành đám tinh thể lớn, mà chúng hình thành và biến mất liên tục theo thời gian ủ nhiệt trong tất cả các vùng thể tích hạt nano. Tuy nhiên tất suất xuất hiện nhân và nhân biến mất diễn ra không đều trong hạt nano. Cụ thể, tần suất xuất hiện nhân mới hình thành và tần suất biến mất của các nhân này xảy ra mạnh nhất trong các vùng 4 và 3 . Đo đó mà đám tinh thể có xu hướng tăng theo hướng từ vùng 4 và 3 tới tâm của hạt nano, nghĩa là tăng từ vùng 4 và 3 vào lõi hạt nano. Tổng hợp những quan sát trên, chúng tôi kết luận rằng, quá trình tinh thể hạt nano Fe VĐH xảy ra ban đầu ở trong vùng gần bề mặt, sau đó các đám tinh thể phát triển theo hướng từ lớp gần bề mặt hướng vào tâm của hạt nano. Cuối cùng đám tinh thể mở rộng trên toàn bộ vùng thể tích hạt nano. Quá trình tinh thể kết thúc, hạt nano Fe tinh thể gồm có hai phần: phần lõi có cấu trúc tinh thể bcc và phần bề mặt có cấu trúc pha trộn giữa pha tinh thể 'bcc biến dạng' và pha VĐH. 


\section{Kết luận}

Trong bài báo này, cấu trúc và quá trình tinh thể hạt nano sắt VĐH đã được nghiên cứu thông qua phân tích HPBXT, theo dõi phân bố số lượng các nguyên tử tinh thể theo thời gian hồi phục trong các lớp hạt nano. Mô phỏng cho thấy, sự tinh thể xảy ra đối với hạt nano Fe VĐH khi được hồi phục trong thời gian dài ở $900 \mathrm{~K}$ và không xảy ra đối với hạt nano $\mathrm{Fe} \mathrm{VĐH}$ ở $300 \mathrm{~K}$ và 500 K. Khi được ủ nhiệt, hạt nano Fe VĐH ở $900 \mathrm{~K}$ chuyển sang cấu trúc tinh thể bcc. Kết thúc quá trính tinh thể hạt nano $\mathrm{Fe}$ gồm: lõi cấu trúc tinh thể bcc và bề mặt cấu trúc VĐH. Cơ chế tinh thể quan sát được phù hợp với lý thuyết nhân cổ điểm. Trong mô phỏng này, quá trinh nhân hóa xảy ra không ngẫu nhiên trong hạt nano mà nó tập trung trong vùng gần bề mặt hạt nano. Đám tinh thể phát triển từ vùng giữa hạt nano, sau đó chuyển đến tâm và cuối cùng mở rộng ra các vùng còn lại. Ở $300 \mathrm{~K}$ và $500 \mathrm{~K}$, đám tinh thể trong hạt nano $\mathrm{Fe} \mathrm{VĐH}$ là quá nhỏ, do đó chúng không thể phát triển thành cấu trúc tinh thể.

\section{Lời cảm ơn}

Nghiên cứu này được tài trợ bởi Quỹ Phát triển khoa học và công nghệ Quốc gia (NAFOSTED), mã số 103.02-2018.312.

\section{Tài liệu tham khảo}

[1] J.D. Honeycutt, C.H. Andersen, Molecular dynamics study of melting and freezing of small Lennard-Jones clusters, Journal of Physical Chemistry 91 (1987) 4950-4963. https://doi.org/ 10.1021/j100303a014.

[2] H. Shin, H.S. Jung, K.S. Hong, J.K. Lee, Crystallization process of $\mathrm{TiO}_{2}$ nanoparticles in an acidic solution, Chemistry letters 33 (2004) 13821383. https://doi.org/10.1246/cl.2004. 1382.

[3] D. Shi, Z. Li, Y. Zhang, X. Kou, L. Wang, J. Wang, J. Li, Synthesis and characterizations of amorphous titania nanoparticles, Nanoscience and Nanotechnology Letters 1 (2009) 165-170. https://doi.org/10.1166/nnl.2009.1037.

[4] D.N. Srivastava, N. Perkas, A. Gedanken, I. Felner, Sonochemical synthesis of mesoporous iron oxide and accounts of its magnetic and catalytic properties, The Journal of Physical Chemistry B 106 (2002) 1878-1883. https://doi. org/10.1021/jp015532w.

[5] N. Zaim, A. Zaim, M. Kerouad, The hysteresis behavior of an amorphous core/shell magnetic nanoparticle, Physica B: Condensed Matter 549 (2018) 102-106. https://doi.org/ 10.1016/j.physb. 2017.10.071.

[6] L. Gao, Q. Zhang, Effects of amorphous contents and particle size on the photocatalytic properties of $\mathrm{TiO}_{2}$ nanoparticles, Scripta materialia 44 (2001) 1195-1198. https://doi.org/ 10. 1016/S1359-6462(01)00681-9.

[7] G. Madras, B.J. McCoy, Kinetic model for transformation from nanosized amorphous $\mathrm{TiO}_{2}$ to anatase, Crystal growth \& design 7 (2007) 250253. https://doi.org/10.1021/cg060272z.

[8] C.I. Wu, J.W. Huang, Y.L. Wen, S.B. Wen, Y.H. Shen, M.Y. Yeh, Preparation of $\mathrm{TiO}_{2}$ nanoparticles by supercritical carbon dioxide, Materials Letters 62 (2008) 1923-1926. https://doi.org/10. 1016/j.matlet.2007.10.043.

[9] C. Pan, P. Shen, S.Y. Chen, Condensation and crystallization and coalescence of amorphous $\mathrm{Al}_{2} \mathrm{O}_{3}$ nanoparticles, Journal of crystal growth 299 (2007) 393-398. https://doi.org/ 10. 1016/j.jcrysgro.2006.12.006.

[10] M. Epifani, E. Pellicer, J. Arbiol, N. Sergent, T. Pagnier, J.R. Morante, Capping ligand effects on the amorphous-to-crystalline transition of CdSe nanoparticles, Langmuir 24 (2008) 11182-11188. https://doi.org/10.1021/la801859z.

[11] P.H. Kien, M.T. Lan, N.T. Dung, P.K. Hung, Annealing study of amorphous bulk and nanoparticle iron using molecular dynamics simulation. International Journal of Modern Physics B 28 (2014) 1450155 (17 page). https:// doi.org/10.1142/S0217979214501550.

[12] V.V. Hoang, N.H. Cuong, Local icosahedral order and thermodynamics of simulated amorphous Fe. Physica B: Condensed Matter 404 (2009) 340346. https://doi.org/10.1016/ j.physb. 2008.10. 057. 